\title{
Translating neuromodulation
}

\author{
To capitalize on progress in neuromodulation, funders and clinicians should promote not only translational \\ research, but also data sharing.
}

A

$t$ the end of last year,

electroconvulsive therapy devices

were reclassified as US Food and

Drug Administration class II "moderate-

risk" products. They now join non-invasive

transcranial magnetic stimulation and

surgically implanted deep brain stimulation

devices as moderate-risk treatment options

for patients with depression, essential

tremor, Parkinson's disease, epilepsy or

obsessive-compulsive disorder whose

symptoms fail to respond to conventional

drug therapy. Despite the increasing range

of neuromodulatory technologies, these

therapies are not yet widely adopted in

clinical practice. The primary obstacle has

been a lack of critical knowledge concerning

mechanisms of action, optimal dose-

response relationships, and how differences

in neural structure and function among

individuals affect device performance.

To ensure that their investments in

neurotechnologies translate into clinical

benefit, funding agencies and institutions

must make a greater commitment to

research that addresses these gaps and

establish open repositories to collate,

store and share data from across studies and subjects.

This issue's Focus explores the ways in which neurotechnologies are making inroads in both the research and clinical settings. Over the past five years, several global initiatives have established large-scale neuroscience collaborations to deepen our understanding of the brain and galvanize neurotechnology development. In the United States, the Brain Research through Advancing Innovative Neurotechnologies (BRAIN) Initiative has received over $\$ 1$ billion in funding and launched more than 500 projects. These have focused on high-throughput imaging, connectomics, sequencing, and phenotyping approaches to produce finely detailed maps of brain tissues and to catalog cellular subtypes. Other efforts have brought together expertise in neural engineering, materials science and software development to create innovative neural recording interfaces, such as neural dust, nanoelectronic meshes or softening electrodes, to complement multisite electrocorticograms and microelectrodes. New types of brain-computer interfaces are being explored that exploit neural recordings for the voluntary control of external devices and prostheses.
A recurring theme in all of these endeavors is the need for a facile means of storing, sharing and analyzing the data emerging from these platforms. This has prompted the creation of open repositories, such as Neurodata Without Borders, that enable investigators around the world to access standardized data formats and metadata in the cloud, along with detailed information on experimental parameters and protocols.

In the clinical realm, devices are shifting from simple preset stimulation patterns to 'closed-loop' models that continuously monitor the state of the neural environment and respond with an appropriate stimulus (e.g., closed-loop devices in epilepsy). Invasive devices clearly carry greater safety concerns than most drugs: patients receiving implanted devices not only risk infection and collateral tissue damage, but also require experienced surgical teams, specialized nursing and a technical support team capable of providing long-term follow up for battery replacement, software updates or device repairs. Devices can also fail after implantation owing to mechanical fatigue of electrodes and problems related to the foreign-body response. Although noninvasive and wearable devices sidestep these problems, most are unable to reach deepbrain structures or achieve reproducible outcomes for brain diseases.

The ongoing challenge for the field is how to perform clinical testing of devices in a systematic manner. With deep brain stimulation, for example, empirical testing in each individual patient is required following device implantation. Because mechanism of action remains murky, there isn't always sufficient impetus or time for clinicians to test parameter space to find out what works best. Most surgeons are seeking to resolve the patient's symptoms as quickly as possible, not to systematically explore all possibilities for optimal device settings.

Unlike drug testing, clinical trials of devices often don't measure outcome against 'target engagement'-in many cases, identifying the exact anatomical site to be targeted and decoding the neural circuitry at those targets remain works in progress. On top of this, individual differences in neural excitability and plasticity-due to genetic polymorphisms and other patient-level factors-mean every case is different. The lack of mechanistic understanding means that negative results remain uninformative and aspects critical to study replication are frequently missed. Often, there is often no consensus in the field as to what constitutes a biologically inactive sham. Many trials also remain underpowered, being pilot projects with just a few patients, and fail to even attempt to correlate stimulus dose with therapeutic response.

All of these factors make systematic progress in this field daunting. It is especially difficult when the universe of specialized clinical teams engaged in neuromodulatory device development remains relatively small. This leads to siloing of research advances within particular clinical centers and makes it difficult for scientists to compare and reproduce each other's data sequestered within institutions. Post-trial follow up is also complicated if patients with implants move across healthcare systems.

One way to address this problem would be to fund open data repositories that collect subject-derived data from over the life cycle of a device, in a unique device identification system that can be linked to electronic health records. This could work for both implanted devices and wearables. Repositories might be created around specific indications-there are already examples, such as the National Sleep Research Resource or Temple University Hospital EEG dataset-or they might be national or even internationally accessible repositories (like Neurodata Without Borders), with standardized data formats, metadata, detailed protocols and parameters.

The BRAIN Initiative's commitment to translational research has so far been underwhelming. In addition to basic and clinical studies, BRAIN and other funders need to devote more resources to the translational studies bridging the gap between bench and bedside. Ultimately, we don't have to understand the entire brain to make clinical applications of neuromodulation more successful. We just need sufficient human data to better understand the effects of brain stimulation.

Published online: 4 September 2019 https://doi.org/10.1038/s41587-019-0263-3 